\title{
Universiteit
}

Leiden

The Netherlands

\section{Observation of two-photon speckle patterns (Appendix)}

Peeters, W.H.; Moerman, J.J.D.; Exter, M.P. van

\section{Citation}

Peeters, W. H., Moerman, J. J. D., \& Exter, M. P. van. (2010). Observation of two-photon speckle patterns (Appendix). Physical Review Letters, 104(17), 173601.

doi:10.1103/PhysRevLett.104.173601

Version: $\quad$ Not Applicable (or Unknown)

License: $\quad$ Leiden University Non-exclusive license

Downloaded from: https://hdl.handle.net/1887/71382

Note: To cite this publication please use the final published version (if applicable). 


\title{
Observation of Two-Photon Speckle Patterns
}

\begin{abstract}
W. H. Peeters, J. J. D. Moerman, and M.P. van Exter
Huygens Laboratory, Leiden University, P.O. Box 9504, 2300 RA Leiden, The Netherlands (Received 24 November 2009; revised manuscript received 16 March 2010; published 27 April 2010)

We report the observation of speckle patterns in quantum correlations within light that is scattered by a disordered medium. The random medium is illuminated with spatially entangled photon pairs, and fourthorder speckle patterns are spatially resolved by two independently scanning detectors. Spatial entanglement gives two-photon speckle a much richer structure than ordinary one-photon speckle. Our experiments demonstrate that two-photon speckle from a surface scatterer and a volume scatterer look entirely different.
\end{abstract}

DOI: 10.1103/PhysRevLett.104.173601

Optical speckle is the random interference pattern that is observed when coherent radiation is scattered by a disordered scattering medium. The phenomenon has been studied widely since the invention of the laser [1]. Textbook studies generally consider the scattered intensity, which can be regarded as the one-photon probability density. Recently, the concept of speckle was theoretically extended to the two-photon probability density, which is observable as the coincidence count rate between two detectors [2]. The idea is to illuminate a random medium with spatially entangled photon pairs, produced via spontaneous parametric down-conversion (SPDC) [3-6], such that a two-photon speckle pattern is formed in the coincidence count rate.

Two-photon speckle is of great interest for the research on multiple scattering of nonclassical waves [2,7-9]. The coincidence count rate is the key observable when studying entangled states in random media, and two-photon speckle directly visualizes the spatial structure of the entanglement in the scattered light. The subject of two-photon speckle is related to a recent observation of spatial quantum correlations in multiply scattered light [9]. In this observation, however, only the spatially integrated power was measured. Until now, the structure within spatial quantum correlations in multiply scattered light has remained unexplored. Furthermore, two-photon speckle is important for the research on two-photon imaging [10-16], in which random media have not been investigated thus far.

In this Letter we report the experimental observation of two-photon speckle. Spatially entangled photon pairs are scattered of random media, and quantum correlations in the scattered light are spatially resolved by two independently scanning detectors in the far field. First, we introduce a theoretical expression for the autocorrelation function of the two-photon speckle pattern. Second, we present experimental two-photon speckle patterns for surface and volume scatterers, and we demonstrate in which respect they are different. Finally, we discuss requirements for the incident light necessary to generate two-photon speckle.
PACS numbers: 42.50.Dv, 42.25.Dd, 42.30.Ms, 42.65.Lm

Theory.-Figure 1 schematically depicts the experimental setup. A static linear scattering medium is illuminated with photon pairs that originate from a low-gain SPDC source pumped by a continuous-wave laser with full spatial coherence. Detection occurs quasimonochromatically in the far field of the scatterer in transmission geometry [17]. Scattered photons are probabilistically separated at a beam splitter, detected by independently scanning singlephoton counters, and correlated by coincidence logic.

The transverse spatial properties of a pure spatially entangled photon pair are described by the two-photon probability amplitude $[3,18]$

$$
A\left(\mathbf{x}_{1}, \mathbf{x}_{2}\right) \equiv\left\langle\operatorname{vac}\left|\hat{E}^{(+)}\left(\mathbf{x}_{1}\right) \hat{E}^{(+)}\left(\mathbf{x}_{2}\right)\right| \Psi\right\rangle,
$$

where $\hat{E}^{(+)}(\mathbf{x})$ is the positive-frequency electric-field operator at transverse position $\mathbf{x}$. The profile $A\left(\mathbf{x}_{1}, \mathbf{x}_{2}\right)$ is the complex probability amplitude for simultaneous detection of one photon at transverse position $\mathbf{x}_{1}$ and the other photon at transverse position $\mathbf{x}_{2}$. The coincidence count rate between two detectors thus scales as $R\left(\mathbf{x}_{1}, \mathbf{x}_{2}\right) \propto$ $\left|A\left(\mathbf{x}_{1}, \mathbf{x}_{2}\right)\right|^{2}$. Below, we refer to $A\left(\mathbf{x}_{1}, \mathbf{x}_{2}\right)$ and $R\left(\mathbf{x}_{1}, \mathbf{x}_{2}\right)$ as the two-photon field and two-photon intensity, respectively. We will express the transverse coordinates $\mathbf{s}_{1,2}$ in the far-field plane in terms of angles $\boldsymbol{\theta}_{1,2} \equiv \mathbf{s}_{1,2} / f_{d}$, where $f_{d}$ is the focal length of the far-field imaging lens (see Fig. 1).

The formation of two-photon speckle in the coincidence count rate occurs via quantum interference in the randomly scattered two-photon field (theory in Ref. [2]). Extending the analysis in Ref. [2], we concentrate on the average twophoton speckle shape, which depends on both detector

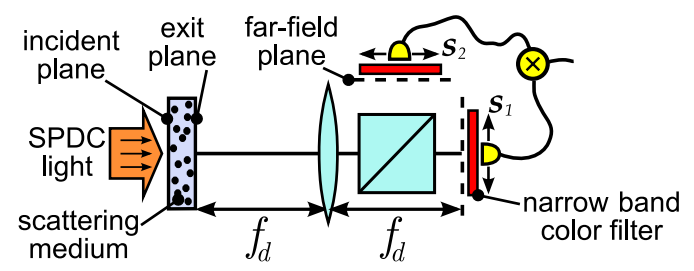

FIG. 1 (color online). Experimental scheme. 
displacements in the far field. The speckle shape is contained in the autocorrelation function of the two-photon speckle pattern

$$
C\left(\boldsymbol{\theta}_{1}, \boldsymbol{\theta}_{2} ; \delta \boldsymbol{\theta}_{1}, \delta \boldsymbol{\theta}_{2}\right) \equiv \overline{R_{\mathrm{FF}}\left(\boldsymbol{\theta}_{1}^{-}, \boldsymbol{\theta}_{2}^{-}\right) R_{\mathrm{FF}}\left(\boldsymbol{\theta}_{1}^{+}, \boldsymbol{\theta}_{2}^{+}\right),}
$$

where $R_{\mathrm{FF}}\left(\boldsymbol{\theta}_{1}, \boldsymbol{\theta}_{2}\right)$ is the coincidence count rate in the farfield plane, and $\boldsymbol{\theta}_{1,2}^{ \pm} \equiv \boldsymbol{\theta}_{1,2} \pm \frac{1}{2} \delta \boldsymbol{\theta}_{1,2}$. The bar denotes averaging over different realizations of disorder.

To arrive at a nice expression for the two-photon speckle autocorrelation function $C\left(\boldsymbol{\theta}_{1}, \boldsymbol{\theta}_{2} ; \delta \boldsymbol{\theta}_{1}, \delta \boldsymbol{\theta}_{2}\right)$, we make two assumptions. First, we assume the opening angle of the scattered light to be much wider than the diffraction angles associated with the average two-photon intensity in the exit plane $\overline{R_{\mathrm{ex}}\left(\mathbf{x}_{1}, \mathbf{x}_{2}\right)}$ (strong-scattering regime). Second, the scattered two-photon field is assumed to exhibit Gaussian statistics [19]. With these assumptions, Eq. (2) can be rewritten as [20]

$$
\begin{aligned}
C\left(\boldsymbol{\theta}_{1}, \boldsymbol{\theta}_{2} ; \delta \boldsymbol{\theta}_{1}, \delta \boldsymbol{\theta}_{2}\right) \approx & \overline{R_{\mathrm{FF}}\left(\boldsymbol{\theta}_{1}, \boldsymbol{\theta}_{2}\right)^{2}} \\
& \times\left[1+\left|\mu^{\prime}\left(\delta \boldsymbol{\theta}_{-}, \delta \boldsymbol{\theta}_{+}\right)\right|^{2}\right],
\end{aligned}
$$

where the speckle shape function $\mu^{\prime}\left(\delta \boldsymbol{\theta}_{-}, \delta \boldsymbol{\theta}_{+}\right)$is expressed in a rotated basis where $\delta \boldsymbol{\theta}_{ \pm} \equiv \frac{1}{\sqrt{2}}\left(\delta \boldsymbol{\theta}_{1} \pm \delta \boldsymbol{\theta}_{2}\right)$. The key result is the speckle shape function

$$
\mu^{\prime}\left(\delta \boldsymbol{\theta}_{-}, \delta \boldsymbol{\theta}_{+}\right)=\frac{\mathfrak{\mho}\left[\overline{R_{\mathrm{ex}}^{\prime}\left(\mathbf{x}_{-}, \mathbf{x}_{+}\right)}\right]\left(k \delta \boldsymbol{\theta}_{-}, k \delta \boldsymbol{\theta}_{+}\right)}{\mathfrak{\mho}\left[\overline{R_{\mathrm{ex}}^{\prime}\left(\mathbf{x}_{-}, \mathbf{x}_{+}\right)}\right](0,0)},
$$

where $\mathfrak{\wp}$ denotes the spatial Fourier transform, and $k$ is the radial wave number of a down-converted photon in vacuum. The average two-photon intensity in the exit plane of the scatterer $\overline{R_{\mathrm{ex}}^{\prime}\left(\mathbf{x}_{-}, \mathbf{x}_{+}\right)}$is expressed in a rotated basis where $\mathbf{x}_{ \pm} \equiv \frac{1}{\sqrt{2}}\left(\mathbf{x}_{1} \pm \mathbf{x}_{2}\right)$, and $\mathbf{x}_{1,2}$ denote transverse positions of the photons in the exit plane.

The theoretical result in Eqs. (3) and (4) shows that the average shape of two-photon speckle spots in the far field is Fourier related to the spatial structure of the average twophoton intensity in the exit plane. Note that the two-photon speckle size can be very different along the difference coordinate $\boldsymbol{\theta}_{1}-\boldsymbol{\theta}_{2}$ and the sum coordinate $\boldsymbol{\theta}_{1}+\boldsymbol{\theta}_{2}$. The size of the speckle spots along the difference coordinate is inversely proportional to the distance between the photons in the exit plane, whereas the size along the sum coordinate depends on the sum coordinate of the photons in the exit plane.

Equations (3) and (4) have a well-known analog in ordinary one-photon speckle. The one-photon speckle theorem assumes spatially coherent illumination, and it states that the shape of one-photon speckle spots in the far field is Fourier related to the average intensity in the exit plane [1]. The analogy is based on similarities between the autocorrelation function of the electric-field amplitude $\overline{E^{*}(\mathbf{x}) E\left(\mathbf{x}^{\prime}\right)}$ for the one-photon case and the autocorrelation function of a pure two-photon probability amplitude $\overline{A^{*}\left(\mathbf{x}_{1}, \mathbf{x}_{2}\right) A\left(\mathbf{x}_{1}^{\prime}, \mathbf{x}_{2}^{\prime}\right)}$ for the two-photon case [21]. The latter function is closely related to the two-photon cross-spectral density function [22]. Both functions mathematically denote two-photon field mixtures, either via a quantum average (in Ref. [22]) or via different realizations of disorder (in our case).

Experimental setup (see Fig. 2).-Photon pairs are generated via SPDC in a cw-pumped collinear type-I geometry in a 5-mm-long periodically poled $\mathrm{KTiOPO}_{4}$ crystal (pump $200 \mathrm{~mW}$ at $413 \mathrm{~nm}$ ). The crystal center is imaged onto the incident plane of the scatterer. Detection occurs via projection onto single-mode fibers that are coupled to photon counters and coincidence logic $\left(\tau_{\text {gate }}=1.73 \mathrm{~ns}\right)$. Spatial resolution in the far-field planes is achieved with tight foci of the detection modes $\left(w_{\text {det }}=140 \mu \mathrm{m}\right)$. When the fiber holders are scanned transversely, the detection modes move in their far-field planes but remain fixed in the exit plane of the scatterer. The detectors are placed behind narrow band spectral filters such that the experiment operates in the quasimonochromatic regime $(5 \mathrm{~nm}$ at $826 \mathrm{~nm}$ ) [17]. The figures in this Letter show coincidence count rates that are corrected for accidental coincidence counts. The generated two-photon field for our source is thoroughly analyzed in Refs. $[23,24]$. The Schmidt number of the spatial entanglement, which quantifies the effective number of independent spatial modes, is $K \approx 83$ [25].

Our experiments are designed to demonstrate the importance of the Fourier relation for the two-photon speckle shape in Eqs. (3) and (4). Three different scatter configurations are chosen (see Fig. 2). Configurations (a) and (b) represent surface scatterers involving a single diffuser. Configuration (c) mimics a volume scatterer comprising two diffusers positioned in one another's far field. A surface scatterer does not directly affect the transverse position of an incident photon. Hence, the two-photon speckle shape will correspond to the strong positional correlations of the incident light. A volume scatterer, on the other hand, is capable of moving a photon transversely during the photon's propagation from the incident plane to the exit plane. As a consequence, the average positional correlation in the exit plane will be smeared out, and the two-photon

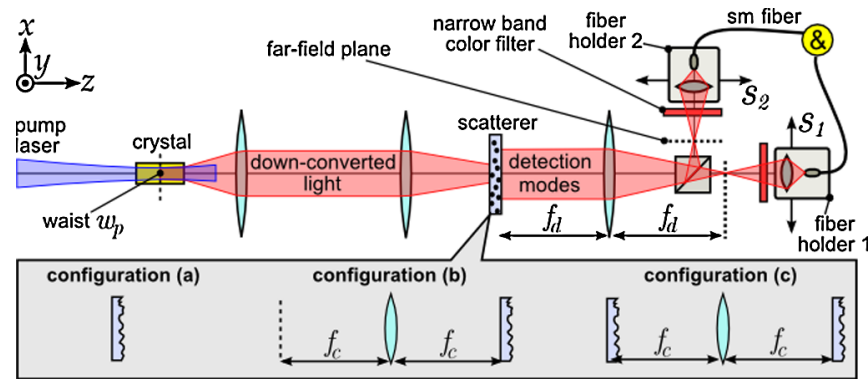

FIG. 2 (color online). Experimental setup. Scattered photons are probabilistically separated at a beam splitter, collected by two single-mode fibers, and detected by photon counters and coincidence logic. Three scatter configurations can be chosen (a)-(c). The full width at half maximum scattering angle of each diffuser is $22 \mathrm{mrad}$. The indicated length scales are $w_{p}=$ $160 \mu \mathrm{m}, f_{c}=10 \mathrm{~mm}$, and $f_{d}=250 \mathrm{~mm}$. 


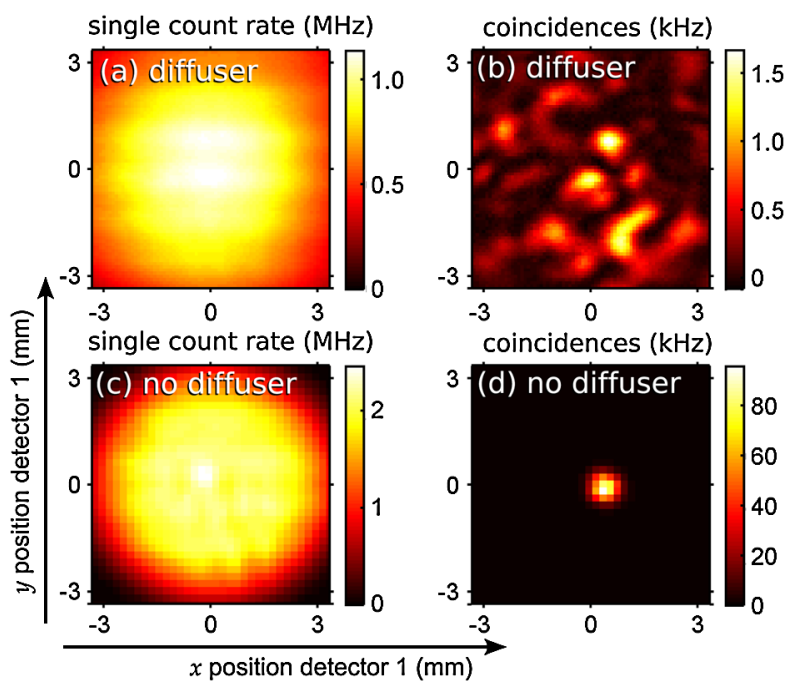

FIG. 3 (color online). (a) Measured single count rate in the far field of a diffuser. (b) Measured coincidence count rate in the same experiment. This two-photon speckle pattern is recorded while keeping the position of detector 2 fixed. Panels (c) and (d) display the reference measurement.

speckle shape is expected to be entirely different. This is remarkable, since in the case of ordinary one-photon speckle, speckle spots are identical for surface and volume scatterers [1].

Experimental results.-First, we discuss an experiment with one scanning and one fixed detector. The experiment is performed in configuration (a) involving a single diffuser. Figures 3(a) and 3(b) show the single count rate and the coincidence count rate, respectively. It is remarkable that speckle is only observed in the coincidence count rate. Because of spatial entanglement, projection of the photon in the fixed detector corresponds to a collapse of the other photon into a speckle pattern. The absence of any ordinary one-photon speckle is consistent with the multimode character of the entangled SPDC light. The reference experiment, in the absence of scattering, is displayed in Figs. 3(c) and 3(d). The sharp peak in the coincidence count rate locates the mirrored position of detector 2 .

Second, we discuss experiments where the positions of both detectors are scanned independently in a horizontal line (see Fig. 4). Only by scanning them both, one gains knowledge about the size of the two-photon speckle spots along both the difference coordinate and the sum coordinate. The two-photon speckle patterns in Figs. 4(a)-4(c) correspond to the three scattering configurations in Fig. 2. All patterns obey mirror symmetry with respect to the diagonal due to the indistinguishability of the photons. Remarkable is the fact that the three patterns look entirely different. The two-photon speckle spots are strongly elongated for the single diffuser [Figs. 4(a) and 4(b)] while the spots are almost isotropic for the volume scatterer [Fig. 4(c)].

In configuration (a) the two-photon source itself is imaged onto the diffuser. The two photons arrive at approximate equal positions on the diffuser since they are created in that way inside the nonlinear crystal. The two-photon intensity is thus tight along the difference coordinate in the plane of the diffuser. In agreement with the Fourier relationship in Eq. (4), two-photon speckle in Fig. 4(a) appears elongated along the difference coordinate. In configuration (b) the far field of the two-photon source is imaged onto the diffuser, and the photons have approximately opposite transverse positions. Accordingly, we observe two-photon speckle spots that are elongated along the sum coordinate in Fig. 4(b). In configuration (c) the second diffuser is illuminated with far-field patterns similar to Fig. 4(a). The average two-photon intensity is now wide in both $x_{+}$and $x_{-}$directions. In accordance, we observe two-photon speckle spots in Fig. 4(c) that are small along both diagonal coordinates.

The orientation of the speckle spots in Figs. 4(a) and 4(b) and the isotropy in Fig. 4(c) can be qualitatively understood via the Klyshko picture of two-photon imaging $[10,26]$. This interpretation follows one detected photon backwards in time to be converted into the second forwardpropagating photon via a virtual reflection at the nonlinear crystal. However, a quantitative understanding of the twophoton speckle size requires detailed knowledge of the spatial and angular limitations of the generating process $[3,4]$; i.e., one needs the explicit expression for the generated two-photon field for our source $[23,24]$.

We now discuss a quantitative analysis of the twophoton speckle size for configuration (a). Seven experimental speckle patterns yield average speckle sizes of $(0.44 \pm 0.05)$ and $(4.4 \pm 0.2) \mathrm{mm}$ along the sum diagonal and difference diagonal, respectively. The theoretical values are 0.41 and $5.4 \mathrm{~mm}$ as calculated from Eqs. (3) and (4)

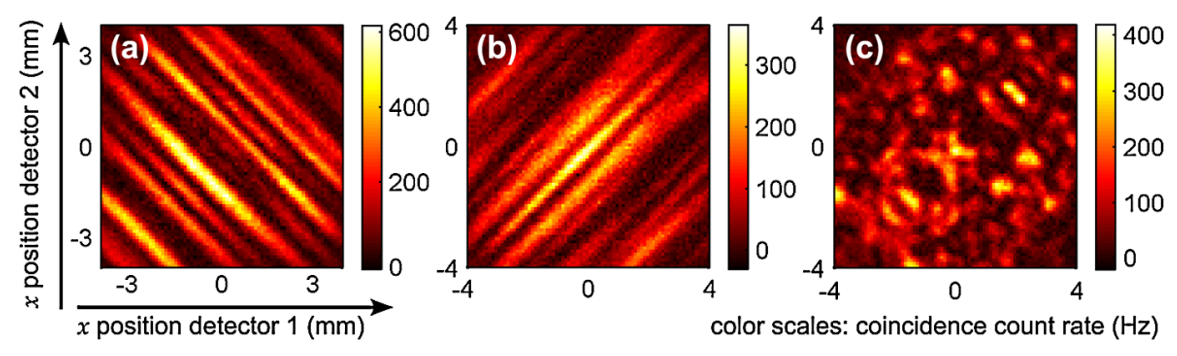

FIG. 4 (color online). Measured two-photon speckle patterns recorded with two independently scanning detectors. The patterns (a)(c) directly correspond to scattering configurations (a)-(c) in Fig. 2. 
and the generated two-photon field $[23,24]$. The confined dimension is in excellent agreement with theory while the elongated size is slightly smaller than expected. We attribute this small disagreement to the fact that our experiment does not fully operate in the strong-scattering regime assumed in Eqs. (3) and (4).

Discussion.-Two-photon speckle becomes interesting only if the incident light contains multimode spatial correlations. If the incident light were in a single spatial mode, any scattered two-photon speckle pattern would factorize into the one-photon intensities via $R\left(\boldsymbol{\theta}_{1}, \boldsymbol{\theta}_{2}\right) \propto I\left(\boldsymbol{\theta}_{1}\right) I\left(\boldsymbol{\theta}_{2}\right)$. This pattern obviously contains the same information as the intensity pattern $I(\boldsymbol{\theta})$.

Correlations in thermal light cannot generate the nonfactorizable features in Fig. 4. Thermal correlations are phase insensitive and are not affected by a diffuser [16]. This is easily understood via the Klyshko-type picture for phase-insensitive correlations, in which the crystal is replaced by a phase-conjugate mirror instead of a real mirror [15]. Two-photon speckle is thus essentially different from ghost imaging because the latter works fine with thermal light $[13,27]$. The difference stems from the fact that in our scheme both photons pass through the sample, whereas in ghost imaging, one of the photons propagates through an empty reference arm. The features in Fig. 4 require phasesensitive correlations in the incident light [16]. These correlations are commonly generated via the nonlinear process of SPDC and are not present in thermal light. Our experiments are performed with nonclassical light where subsequent entangled photon pairs can be temporally resolved [16].

The presence of the nonfactorizable features in Fig. 4 demonstrates that two-photon speckle has a richer structure than ordinary one-photon speckle. Because of the spatial entanglement between the photons, projection of one of the photons corresponds to a full collapse of the other photon into a speckled mode profile [see Fig. 3(b)]. The dimensionality of the entanglement quantifies the effective number of different projected one-photon speckle patterns. If someone wishes to generate all these speckle patterns with a coherent laser beam, she or he will have to adjust the mode profile of the incident beam many times. Spatial entanglement allows one to obtain many projected onephoton speckle patterns without changing the geometry of the incident light.

Conclusion.-We have observed two-photon speckle patterns in the far field of disordered scattering media. Spatial entanglement of the incident light gives two-photon speckle a much richer structure than ordinary one-photon speckle. Our work paves the way for future research on multiple scattering of entangled photons. It would be interesting to establish the connection with universal conductance fluctuations [28] and enhanced backscattering of light [29].

We thank J.P. Woerdman, T. Kauffmann, and H. Di Lorenzo Pires for useful discussions. This research was supported by the Dutch Science Foundation NWO/
FOM and the EU under the FET-Open Agreement "HIDEAS" No. FP7-ICT-221906.

[1] J.W. Goodman, Speckle Phenomena in Optics (Roberts and Company, Greenwood Village, CO, 2007).

[2] C. W. J. Beenakker, J.W.F. Venderbos, and M.P. van Exter, Phys. Rev. Lett. 102, 193601 (2009).

[3] M. H. Rubin, Phys. Rev. A 54, 5349 (1996).

[4] C.H. Monken, P.H. Souto Ribeiro, and S. Pádua, Phys. Rev. A 57, 3123 (1998).

[5] Y. Shih, Rep. Prog. Phys. 66, 1009 (2003).

[6] A. Gatti et al., Phys. Rev. Lett. 102, 223601 (2009).

[7] P. Lodahl, A. P. Mosk, and A. Lagendijk, Phys. Rev. Lett. 95, 173901 (2005).

[8] S. E. Skipetrov, Phys. Rev. A 75, 053808 (2007).

[9] S. Smolka et al., Phys. Rev. Lett. 102, 193901 (2009).

[10] D. N. Klyshko, Zh. Eksp. Teor. Fiz. 94, 82 (1988) [Sov. Phys. JETP 67, 1131 (1988)].

[11] D. V. Strekalov et al., Phys. Rev. Lett. 74, 3600 (1995).

[12] B. E. A. Saleh et al., Phys. Rev. A 62, 043816 (2000).

[13] A. Gatti et al., Phys. Rev. Lett. 93, 093602 (2004).

[14] B.E. A. Saleh, M. C. Teich, and A. V. Sergienko, Phys. Rev. Lett. 94, 223601 (2005).

[15] G. Scarcelli, V. Berardi, and Y. Shih, Appl. Phys. Lett. 88, 061106 (2006).

[16] B. I. Erkmen and J. H. Shapiro, Phys. Rev. A 78, 023835 (2008).

[17] By quasimonochromatic we mean that the optical detection bandwidth $\Delta \nu$ is small in comparison with $\tau_{\text {scat }}^{-1}$, where $\tau_{\text {scat }}$ is the spread in scattering times. The twophoton speckle contrast is expected to diminish for large bandwidth since the spatial electric-field propagator typically varies over frequency intervals $\tau_{\text {scat }}^{-1}$.

[18] The subpicosecond structure in the $t_{2}-t_{1}$ coordinate of the two-photon probability amplitude is left out because spatial-temporal correlations [6] will be absent within the applied optical detection bandwidth $\Delta \nu$ that is much smaller than the phase-matching bandwidth.

[19] This assumption is valid for high-dimensional entanglement and becomes approximate for small Schmidt numbers (see Fig. 2 in Ref. [2]).

[20] See supplementary material at http://link.aps.org/ supplemental/10.1103/PhysRevLett.104.173601 for details of the derivation.

[21] This analogy is different from a previously investigated duality between the first-order coherence function and the two-photon probability amplitude [12-14].

[22] A. K. Jha and R.W. Boyd, Phys. Rev. A 81, 013828 (2010).

[23] W. H. Peeters et al., Phys. Rev. A 79, 043817 (2009).

[24] H. Di Lorenzo Pires and M. P. van Exter, Phys. Rev. A 80, 053820 (2009).

[25] H. Di Lorenzo Pires, C. H. Monken, and M. P. van Exter, Phys. Rev. A 80, 022307 (2009).

[26] D. N. Klyshko, Phys. Lett. A 132, 299 (1988).

[27] A. Valencia et al., Phys. Rev. Lett. 94, 063601 (2005).

[28] F. Scheffold and G. Maret, Phys. Rev. Lett. 81, 5800 (1998).

[29] M. P. Van Albada and A. Lagendijk, Phys. Rev. Lett. 55, 2692 (1985). 